\title{
Anesthetic management of burn-associated dilated cardiomyopathy with difficult intubation for bilateral correction of ectropion
}

\author{
Nasrullah Sheikh ${ }^{1}$, Muhammad Salik ${ }^{2}$, Ahmed Faraz ${ }^{3}$, Mohamed Makhloof Aldahish ${ }^{4}$ \\ Affiliations: \\ 1, 4. Department of Anesthesiology, Security Forces Hospital, P.O. Box 3643, Riyadh 11481, Kingdom of Saudi Arabia. \\ 2, 3. Alfaisal University, College of Medicine, P. O. Box 50927, Riyadh 11533, Kingdom of Saudi Arabia. \\ Correspondence: Dr. Nasrullah Sheikh; E-mail: Nasrullahok@gmail.com
}

\section{Abstract}

Anesthetic management of patients with dilated cardiomyopathy (DCM) can be challenging for anesthesiologists because it is associated with high perioperative mortality rate.

This case report describes successful management and important anesthetic considerations in a 15-year-old boy, a known case of burn-associated dilated cardiomyopathy, who underwent bilateral ectropion correction surgery. In such patients, it is imperative to have meticulous preoperative planning, wise selection of medications, and tailored anesthetic technique in order to achieve a favourable outcome.

Key words: Anesthesia; Burns; Cardiomyopathy, Dilated; Ketamine; Propofol; Dopamine

Citation: Sheikh N, Salik M, Faraz A, Aldahish MM. Anesthetic management of burn-associated dilated cardiomyopathy with difficult intubation for bilateral correction of ectropion. Anaesth. pain intensive care 2020;24(5):552-554

Received: 22 August 2020, Reviewed: 23 August 2020, Accepted: 28 August 2020

\section{Introduction}

Dilated cardiomyopathy (DCM) is characterized by left ventricular or biventricular dilation which significantly reduces myocardial contractility. ${ }^{1}$ It is a very rare complication in patients with burn injury and the underlying pathophysiology is thought to be because of relative myocardial ischemia due to the hypermetabolic state caused by burn injuries. ${ }^{2}$ Anesthetic management in such cases is challenging because of poor cardiac output and the increased risk of arrhythmias and sudden cardiac death. ${ }^{3}$ There is a limited amount of literature available for anesthetic management in burn-related cardiomyopathy with reduced ejection fraction (EF) and difficult intubation; therefore, we present this case report.

\section{Case Report}

A 15-year-old boy, known case of burn-associated dilated cardiomyopathy, presented to the operating room (OR) for correction of bilateral ectropion to prevent blindness from exposure keratopathy due to severe facial contractures after burn injuries.

In background history, he suffered a $45 \%$ fullthickness burn along with inhalational injury and was intubated in the emergency department and shifted to the intensive care unit (ICU). During the ICU stay, he underwent multiple surgeries for fullthickness skin grafting under general anesthesia (GA) without any complications. He had difficulty in weaning off the ventilator, therefore he underwent tracheostomy. Later on, cardiology consultation was requested for his persistent tachycardia and the echocardiography report revealed severely dilated left ventricle (LV) and profound LV systolic dysfunction with an EF of $10 \%$. Thus, he was diagnosed as a case of burn-associated dilated cardiomyopathy, and after seven weeks his tracheostomy was reversed. Subsequently, he was discharged with the following medications: bisoprolol $2.5 \mathrm{mg} \mathrm{BD}$, lisinopril $5 \mathrm{mg} \mathrm{OD}$, digoxin 
$125 \mu \mathrm{g}$ OD, ivabradine $5 \mathrm{mg} \mathrm{BD}$ and furosemide 20 mg BD.

During preoperative anesthesia assessment, his height was $154 \mathrm{~cm}$ and weighed $48 \mathrm{~kg}$ with a body mass index of 20.2. His vitals were: heart rate (HR) 85 beats/min, blood pressure (BP) 100/55 mmHg, $\mathrm{SpO}_{2} \quad 97 \%$ on room air with Functional Classification II. On physical examination, his chest was clear and there were no signs suggestive of congestive heart failure and the rest of the examination was unremarkable. All routine laboratory investigations were within normal limits. Upon airway assessment, he had a difficult airway with two fingers mouth opening and restricted neck extension due to severe contractures in the face and neck region. He was labeled as American Society of Anesthesiologist (ASA) class IV. Local anesthesia was strongly refused by the patient and the surgeon due to the complex nature of the surgery. Therefore, the risks and benefits of surgery under GA were thoroughly discussed with the family and a high-risk consent was obtained and the ICU bed was arranged. Our anesthesia plan was to tackle the low EF with dopamine infusion before induction and ketofol was chosen as an induction agent along with a fiberoptic bronchoscope for tracheal intubation.

The patient was brought to the OR premedicated with IV midazolam $(1.5 \mathrm{mg})$ and was attached to ASA standard monitoring: ECG, NIBP, $\mathrm{SpO}_{2}$, $\mathrm{EtCO}_{2}$, temperature, and nerve stimulator. Additionally, the arterial line was inserted under local anesthesia. His vitals were recorded as HR 80 beats/min, BP 92/53 mmHg, and $\mathrm{SpO}_{2} 100 \%$. Before induction of anesthesia, dopamine infusion at $5-10$ $\mu \mathrm{g} / \mathrm{kg} / \mathrm{m}$ was commenced. The patient was preoxygenated and induced with titrated doses of ketofol (ketamine $40 \mathrm{mg}$ and propofol $40 \mathrm{mg}$ ) in a controlled manner. Subsequently, bag-mask ventilation was confirmed and muscle relaxant (cisatracurium $7 \mathrm{mg}$ ) was given. Tracheal intubation was performed in the first attempt using a fiber optic bronchoscope. The patient was switched to the anesthesia machine on SIMV mode and with tidal volume of $6 \mathrm{ml} / \mathrm{kg}$ and respiratory rate of 12 breaths/min without application of positive endexpiratory pressure. Anesthesia was maintained using desflurane in $50 \%$ oxygen/air at the minimum alveolar concentration (MAC) of 0.6 - 0.8 along with boluses of $30 \mu \mathrm{g}$ fentanyl every $30 \mathrm{~min}$. Intra-venous Ringer's lactate was cautiously infused at the rate of $90 \mathrm{ml} / \mathrm{hr}$. Moreover, intraoperative arterial blood gases were recorded as follows: $\mathrm{pH} 7.38, \mathrm{pCO}_{2} 32$
$\mathrm{mmHg}, \mathrm{pO}_{2} 108 \mathrm{mmHg}, \mathrm{HCO}_{3} 20.7 \mathrm{mEq} / \mathrm{L}$, and Base excess $-5 \mathrm{mEq} / \mathrm{L}$. After three hours of uneventful surgery and vigilant anesthesia monitoring, the patient was extubated when fully awake and shifted to the post-anesthesia care unit (PACU).

In PACU, he received $5 \mathrm{mg}$ boluses of meperidine (a total of $40 \mathrm{mg}$ ) for post-operative pain management. He was closely observed for two hours and he remained vitally stable. Thereafter, he was transferred to ICU and subsequently discharged home after one week.

\section{Discussion}

Induction of anesthesia is the most critical phase in a patient with extremely low EF, especially when combined with difficult intubation. In such cases of DCM, the key anesthetic management goals are to maintain myocardial contractility, avoid cardiacdepressant drugs, prevent increase in afterload, and avoid volume overload. ${ }^{4}$

Etomidate has been considered a choice of induction agent in patients with reduced cardiac output due to its minimal cardio-depressive effects. However, a previous study concluded that etomidate increases LV afterload, which further compromises cardiac output especially in patients with pre-existing reduced LV function. ${ }^{5}$ While, Ketamine also increases peripheral vascular resistance (PVR) due to its sympathetic activity, but it can be safely used in combination with propofol, midazolam, or fentanyl in patients with impaired cardiac function. ${ }^{6}$ As propofol reduces PVR thereby it counteracts the sympathetic effect of ketamine. Thus, titrated doses of ketofol (ketamine $40 \mathrm{mg}$ and propofol $40 \mathrm{mg}$ ) were used to induce the anesthesia to avoid the wide fluctuations in hemodynamics.

Volatile anesthetic agents are typically cardiodepressant; however, it does not affect the cardiac contractility when used in combination with fentanyl $(2-3 \mu \mathrm{g} / \mathrm{kg})$ at lower MAC range (0.5-1.0). ${ }^{3}$ Thus, in our patient, anesthesia was maintained using desflurane at the MAC of $0.6-0.8$ along with boluses of $30 \mu \mathrm{g}$ fentanyl every $30 \mathrm{~min}$.

\section{Conflict of interest}

Nil declared by the authors

\section{Funding}

No funding was received for this paper 


\section{Authors' contribution}

NS: Concept, design, literature search, manuscript preparation and review, guarantor MS, AF: Design, literature search, manuscript preparation, editing \& review

MA: Concept, design, manuscript editing \& review

\section{References}

1. Dec GW, Fuster V. Idiopathic dilated cardiomyopathy. N Engl J Med. 1994;331(23):1564$1575 . \quad$ [PubMed] DOl: 10.1056/NEJM199412083312307

2. Fukui R, Suzuki H, Miyagawa $N$, Endo $T$, Kaneta $T$, Sugimura K, et al. Burn-associated delayed dilated cardiomyopathy evaluated by cardiac PET and SPECT: Report of a case. J Cardiol Cases. 2014;10(5):180-183. [PubMed] DOI: 10.1016/ij.jcase.2014.07.005
3. Chen CQ, Wang X, Zhang J, Zhu SM. Anesthetic management of patients with dilated cardiomyopathy for noncardiac surgery. Eur Rev Med Pharmacol Sci. 2017;21:627-634. [PubMed]

4. Thiagarajah $\mathrm{PH}$, Thiagarajah S, Frost EA. Anesthetic considerations in patients with cardiomyopathies - A review. Middle East J Anaesthesiol. 2009;20:347354. [PubMed]

5. Pagel PS, Hettrick DA, Kersten JR, Tessmer JP, Lowe $D$, Warltier DC. Etomidate adversely alters determinants of left ventricular afterload in dogs with dilated cardiomyopathy. Anesth Analg. 1998;86(5):932-928. [PubMed] DOl: 10.1097/00000539-199805000-00003

6. Yamaguchi S, Wake K, Mishio M, Okuda Y, Kitajima $\mathrm{T}$. Anesthetic management of a patient with dilated cardiomyopathy under total intravenous anesthesia with propofol and ketamine combined with continuous epidural analgesia. Masui. 1999;48(11):1232-1234. [PubMed] 